Real Analysis Exchange

Vol. 24(2), 1998/9, pp. 807-814

Marcin Szyszkowski* Department of Mathematics, West Virginia University, Morgantown, WV 26506-6310; e-mail: Marcin@math.wvu.edu

\title{
POINTS OF WEAK SYMMETRIC CONTINUITY
}

\begin{abstract}
We show that every set of reals is a set of points of weak symmetrical continuity for some function and compare it with other generalized continuities. We also make some remarks on points of weak symmetric continuity when function is finite valued.
\end{abstract}

Our terminology is standard and follows [2]. Let us recall a known fact about points of continuity. (See e.g. [6].)

Fact 1 . The set of points of continuity of any function (from $\mathbb{R}$ to $\mathbb{R}$ ) is a $G_{\delta}$ set and any $G_{\delta}$ set is the set of points of continuity for some function.

Many authors have investigated what happens if we replace the ordinary continuity by other types of continuity, i.e., which sets may be obtained as sets of points of different types of generalized continuity. First let us look at the symmetric continuity.

Definition 2. A function $f: \mathbb{R} \rightarrow \mathbb{R}$ is symmetrically continuous at a point $x$ if

$$
\forall_{\varepsilon>0} \exists_{\delta>0} \forall_{h<\delta} \quad|f(x+h)-f(x-h)|<\varepsilon .
$$

Symmetrical continuity is obviously a weaker notion than ordinary continuity and Fact 1 is not valid any more for points of symmetric continuity. In fact it is an old problem of Marcus to characterize these points. (For partial results see Thomson [8] and Jaskuła, Szkopińska [5].)

Another notion we consider is weak continuity.

Key Words: symmetric continuity

Mathematical Reviews subject classification: Primary 26A15, Secondary 26A03

Received by the editors April 2, 1998

* This paper was written under supervision of K. Ciesielski. The author wishes to thank him for many helpful conversations. 
Definition 3. A function $f: \mathbb{R} \rightarrow \mathbb{R}$ is weakly continuous at a point $x$ if there exist the sequences $\left\langle x_{n}\right\rangle$ and $\left\langle y_{n}\right\rangle$ such that $x_{n} \nearrow x, y_{n} \searrow x$, and

$$
\lim _{n \rightarrow \infty} f\left(x_{n}\right)=\lim _{n \rightarrow \infty} f\left(y_{n}\right)=f(x) .
$$

In particular $f\left(x_{n}\right)-f\left(y_{n}\right) \rightarrow 0$.

The next, rather unexpected, theorem says that weak continuity is indeed a very weak notion.

Theorem 4. [9, Chap. 2] Every function $f: \mathbb{R} \rightarrow \mathbb{R}$ is weakly continuous everywhere except a countable set and every countable subset of $\mathbb{R}$ is the set of points of weak discontinuity for some function.

Proof. For the first part see for example [9, Chap. 2].

For the second part, let $A=\left\{a_{n}: n \in \omega\right\}$ be a countable set. The function $f(x)=\sum_{a_{n}<x} 2^{-n}$ is the one we are looking for. Indeed, if $x>a_{n}>y$ then $f(x)-f(y) \geq 2^{-n}$, so $f$ is not weakly continuous at any $a_{n}$. If $x \notin A$ and if $y$ is closer to $x$ than any of the points $a_{1}, \ldots, a_{k}$ then $|f(y)-f(x)| \leq$ $\sum_{n=k+1}^{\infty} 2^{-n}=2^{-k}$ since we do not have any $a_{n}$ 's in $(x-|x-y|, x+|x-y|)$ with $n \leq k$. Therefore $f$ is continuous (so weakly continuous) at $x \notin A$.

Now if we replace weak continuity by its symmetric counterpart, we obtain the following notion.

Definition 5. A function $f: \mathbb{R} \rightarrow \mathbb{R}$ is weakly symmetrically continuous at a point $x$ if there exists a sequence $\left\langle h_{n}\right\rangle$ converging to 0 such that

$$
\lim _{n \rightarrow \infty} f\left(x+h_{n}\right)-f\left(x-h_{n}\right)=0 .
$$

(Paradoxically, weak symmetric continuity is a stronger notion than weak continuity.) We will investigate the problem of characterizing the sets of points where a function can be weakly symmetrically continuous. The first result in this direction was obtained by Ciesielski and Larson, and it looks surprising in light of Theorem 4.

Theorem 6. (Ciesielski, Larson [3]) There is a function $g: \mathbb{R} \rightarrow \mathbb{N}$ that is weakly symmetrically discontinuous everywhere.

The next theorem, generalizing Theorem 6, answers the problem completely.

Theorem 7. Any set of reals is the set of points of weak symmetrical continuity for some function $f: \mathbb{R} \rightarrow \mathbb{N}$. 
Note that since the existence of weakly symmetrically discontinuous function from $\mathbb{R}$ to $\mathbb{R}$ cannot be proved without the Axiom of Choice [3, Cor. 1.5], the same can be said about Theorem 7 .

The main part of the proof is in the following lemma.

Lemma 8. For any set $A \subset \mathbb{R}$ there exists a set $X$ such that

(a) for every $a \in A$ there is a sequence $\left\langle h_{n}\right\rangle$ with $h_{n} \nearrow 0$ and $a \pm h_{n} \in X$;

(b) for every $b \in \mathbb{R} \backslash A$ there is no such a sequence.

Before we start the proof let us remark that the set $X$ may be always chosen to be meager and of measure zero. This partially answers the question stated at the Miniconference in Auburn in 1997 after the presentation of Lemma 8.

The set $X$ can be found as a subset of some special meager and measure zero set $C$. To make the below proof work, we need only that for all $x \in \mathbb{R}$ and for all $n \in \omega$ there are continuum many pairs $y, z \in C \cap\left(x-\frac{1}{n}, x+\frac{1}{n}\right)$ with $\frac{y+z}{2}=x$. Corollary 10 guarantees the existence of such set $C$. In the proof below however, for the sake of simplicity (and since we do not need that strengthened version to prove Theorem 7 ), we take $C=\mathbb{R}$. The reader may notice that with no changes the proof works for other sets $C$.

Proof of Lemma 8. We will construct the set $X$ inductively, using transfinite induction. First note that (a) is equivalent to the following condition.

$\left(\mathrm{a}^{\prime}\right)$ For every $a \in A$ there are the sequences $\left\langle x_{n}\right\rangle$ and $\left\langle y_{n}\right\rangle$ from $X$ such that $x_{n} \nearrow a, y_{n} \searrow a$, and $\frac{x_{n}+y_{n}}{2}=a$ for every $n$.

Condition (b) than says that there are no such sequences $\left\langle x_{n}\right\rangle,\left\langle y_{n}\right\rangle$ for $b \notin A$.

Let $\kappa$ be the cardinality of $A$ and let $A=\left\{a_{\alpha}: \alpha<\kappa\right\}$ be an enumeration of $A$. By induction on $\alpha<\kappa$ we construct the sequences $\left\langle x_{n}^{\alpha}: n<\omega\right\rangle$ and $\left\langle y_{n}^{\alpha}: n<\omega\right\rangle$ witnessing $\left(\mathrm{a}^{\prime}\right)$ for $a=a_{\alpha}$, aiming for $X=\bigcup_{\alpha<\kappa}\left\{x_{n}^{\alpha}, y_{n}^{\alpha}: n<\omega\right\}$. The challenge will be in maintaining condition (b). We will use the following auxiliary sets:

$$
\begin{gathered}
X_{\alpha}=\bigcup_{\beta<\alpha}\left(\left\{x_{n}^{\beta}: n<\omega\right\} \cup\left\{y_{n}^{\beta}: n<\omega\right\}\right), \\
B_{\alpha}=\left\{\frac{x+y}{2}: x, y \in X_{\alpha}\right\} \backslash A,
\end{gathered}
$$

and the functions $f_{\alpha}: B_{\alpha} \rightarrow \mathbb{R}$ defined by

$$
f_{\alpha}(b)=\inf \left\{|b-x|: x \in X_{\alpha} \text { and } \exists_{y \in X_{\alpha}} \frac{x+y}{2}=b\right\} .
$$


(Here $|b-x|$ denotes the distance between the numbers $b$ and $x$.)

In the construction we will maintain the following two inductive conditions:

(c) $f_{\alpha}(x)>0$ for each $x \in B_{\alpha}$, and

(d) $f_{\beta} \subset f_{\alpha}$ for $\beta \leq \alpha$.

Conditions (c) and (d) imply that (b) holds for every point in $\bigcup_{\alpha<\kappa} B_{\alpha}$, since $f=\bigcup_{\alpha<\kappa} f_{\alpha}$ is positive on $\bigcup_{\alpha<\kappa} B_{\alpha}$. Hence, maintaining (c) and (d) during the construction will imply that (b) holds.

For every point outside $\bigcup_{\alpha<\kappa} B_{\alpha}$, condition (b) is automatically satisfied and the set $X$ will have the desired property.

Take the first sequence $\left\langle x_{n}^{0}\right\rangle$ to be $x_{n}^{0}=a_{0}+\frac{1}{n}$. Then $y_{n}^{0}=a_{0}-\frac{1}{n}$, $X_{0}=\left\{x_{n}^{0}, y_{n}^{0}: n<\omega\right\}$, and $B_{0}=\left\{\frac{x+y}{2}: x, y \in X_{0}\right\} \backslash A$. Since the only accumulation point of $X_{0}$ is $a_{0} \in A$ then $f_{0}(x)>0$ for all $x \neq a_{0}$ and (c) is satisfied.

Assume that for some $\alpha<\kappa$ we have already defined the sequences $\left\langle x_{n}^{\beta}\right\rangle$ and $\left\langle y_{n}^{\beta}\right\rangle$ for all $\beta<\alpha$. We choose sequences $\left\langle x_{n}^{\alpha}\right\rangle$ and $\left\langle y_{n}^{\alpha}\right\rangle$ satisfying $\left(\mathrm{a}^{\prime}\right)$ so that

$$
\forall_{b \in B_{\beta}} \forall_{n} 2 b-x_{n}^{\alpha} \notin \bigcup_{\beta<\alpha} X_{\beta} \cup\left\{x_{k}^{\alpha}, y_{k}^{\alpha}: k<n\right\}
$$

and

$$
\forall_{b \in B_{\beta}} \forall_{n} 2 b-y_{n}^{\alpha} \notin \bigcup_{\beta<\alpha} X_{\beta} \cup\left\{x_{k}^{\alpha}, y_{k}^{\alpha}: k<n\right\} .
$$

Such sequences exist since we may choose points $x_{n}^{\alpha}$ and $y_{n}^{\alpha}$ inductively so that

$$
x_{n}^{\alpha}, y_{n}^{\alpha} \notin\left\{2 b-x: b \in \bigcup_{\beta<\alpha} B_{\beta} \& x \in\left(\bigcup_{\beta<\alpha} X_{\beta} \cup\left\{x_{k}^{\alpha}, y_{k}^{\alpha}: k<n\right\}\right)\right\}
$$

(that set has cardinality $\left|\bigcup_{\beta<\alpha} B_{\beta}\right| \otimes\left|\bigcup_{\beta<\alpha} X_{\beta}\right| \leq|\alpha| \otimes \omega<\mathfrak{c}$ ) and $\frac{x_{n}+y_{n}}{2}=$ $a_{\alpha}$. It is clear that both inductive conditions are preserved.

Now putting $X=\bigcup_{\alpha<\kappa} X_{\alpha}=\bigcup_{\alpha<\kappa}\left\{x_{n}^{\alpha}, y_{n}^{\alpha}: n<\omega\right\}$, we see that (a) is obviously satisfied and (b) is satisfied since $f=\bigcup_{\alpha<\kappa} f_{\alpha}$ is a positive function and for every point $b$ not in $A$ either we do not have a pair of sequences contained in $X$ and symmetric about that point or these sequences cannot have elements closer to $b$ than $f(b)$.

Proof of Theorem 7 . Let $g: \mathbb{R} \rightarrow \mathbb{N}$ be an everywhere weakly symmetrically discontinuous function and $A \subset \mathbb{R}$. We'll construct a function $f$ that 
has points of weak symmetric continuity exactly in the set A. Let X be the set from Lemma 8 for the set $\mathrm{A}$. We define

$$
f(x)= \begin{cases}g(x)+1 & \text { for } x \notin X \\ 0 & \text { for } x \in X .\end{cases}
$$

Obviously every point of $\mathrm{A}$ is a point of weak symmetric continuity of $f$. (There is a symmetric sequence in $\mathrm{X}$ converging to this point.) If $x \notin A$ then we do not have a sequence symmetrically convergent to $x$ contained in $X$. We also do not have such a sequence contained in $\mathbb{R} \backslash X$ satisfying the definition of weak symmetric continuity for $x$, because $g$ is weakly symmetrically discontinuous everywhere. Finally any "mixed" sequence will not satisfy that definition either since $f$ differ on points from $X$ and outside of $X$ by at least 1 .

Now we provide the proof of the existence of measure zero and meager set $C$. Since the main theorems of this paper do not require that set, the reader may skip that part. The author includes this since it seems to him that it is worth adding that "small" sets can have big "weak symmetric closure." Note also, that the property stated in Theorem 9 is closely related to the Steinhaus property that every number in $[0,1]$ is the sum of two numbers from the Cantor ternary set.

We will use the base 7 expansion, i.e., for $x \in[0.1]$ we write $x=0 . x_{1} x_{2} \ldots$ meaning that $x=\sum_{i=1}^{\infty} \frac{x_{i}}{7^{i}}, x_{i} \in\{0,1,2,3,4,5,6\}$.

For every finite sequence $x_{1} x_{2} \ldots x_{n}$, where $x_{i} \in\{0,1,2,3,4,5,6\}$, let

$$
C_{x_{1} x_{2} \ldots x_{n}}=\left\{x \in[0,1]: x=0 . x_{1} x_{2} \ldots x_{n} x_{n+1} \ldots x_{i} \neq 3 \text { for } i>n\right\} .
$$

$C_{x_{1} x_{2} \ldots x_{n}}$ is a "Cantor $\frac{1}{7}$ " set. It is a perfect set of measure zero (thus, nowhere dense).

Denote also

$$
\hat{C}=\bigcup\left\{C_{x_{1} x_{2} \ldots x_{n}}: n \in \omega\right\}
$$

The set $\hat{C}$ being a countable union of measure zero and nowhere dense sets has measure zero and is meager. The next theorem says it is the set we are looking for. We say that a set $A \subset \mathbb{R}$ is symmetric about a point $x \in \mathbb{R}$ if $x+h \in A \Longleftrightarrow x-h \in A \forall_{h}$ (the same as $A=2 x-A$ ).

Theorem 9. For every $x \in(0,1)$ and for every open neighbourhood $U$ of $x$ there is a perfect set $P$ contained in $\hat{C} \cap U$ and symmetric about $x$.

Proof. Let $x \in(0,1)$ and let $0 . x_{1} x_{2} \ldots$ be the expansion of $x$. We assume that not almost all $x_{i}^{\prime} s$ are equal to 6 . Let also $U$ be an arbitrary neighbourhood 
of $x$. We fix number $n \in \omega$ such that for all $y=0 . y_{1} y_{2} \ldots$ with $y_{i}=x_{i}$ for $i \leq n$ we have $y \in U$.

We consider three cases.

Case 1. In the sequence $x_{1} x_{2} \ldots$ we have infinitely many 1's or 2's or 3's or 4's or 5's.

Say at first that we have infinitely many 1 's and that $x_{i_{j}}=1, j=1,2,3 \ldots$ and $i_{j}>n$. We define numbers $y$ and $z$.

$y=0 . y_{1} y_{2} \ldots$ and $z=0 . z_{1} z_{2} \ldots$ where

$y_{i}=x_{i}$ for $i \leq n, z_{i}=x_{i}$ for $i \leq n$,

$y_{i_{j}}=0$ or $y_{i_{j}}=1, z_{i_{j}}=2-y_{i_{j}}$,

$y_{i}=2$ if $x_{i}=3, i>n, z_{i}=4$ if $x_{i}=3, i>n$,

$y_{i}=x_{i}, z_{i}=x_{i}$ for all other $i$.

Clearly all such $y$ 's form a "Cantor-like" perfect set and so do $z$ 's, every $y$ and $z$ belongs to $U \cap C_{x_{1} x_{2} \ldots x_{n}}$. For every pair of corresponding $y$ and $z$ we have $\frac{y_{i}+z_{i}}{2}=x_{i}$ so $\frac{y+z}{2}=x$.

If we have infinitely many other digits instead then we repeat the above construction changing only $y_{i_{j}}$ and $z_{i_{j}}$ according to the following formulas.

$x_{i_{j}}=2 \rightarrow y_{i_{j}}=0$ or $y_{i_{j}}=4, z_{i_{j}}=4-y_{i_{j}}$,

$x_{i_{j}}=3 \rightarrow y_{i_{j}}=0$ or $y_{i_{j}}=6, z_{i_{j}}=6-y_{i_{j}}$,

$x_{i_{j}}=4 \rightarrow y_{i_{j}}=2$ or $y_{i_{j}}=4, z_{i_{j}}=8-y_{i_{j}}$,

$x_{i_{j}}=5 \rightarrow y_{i_{j}}=5$ or $y_{i_{j}}=6, z_{i_{j}}=10-y_{i_{j}}$.

Once again we see that $\frac{y_{i}+z_{i}}{2}=x_{i}$ so $\frac{y+z}{2}=x$ and that $y$ 's and $z$ 's form a symmetric perfect set around $x$ that is contained in $\hat{C} \cap U$.

Case 2. In $x_{1} x_{2} \ldots$ we have infinitely many 0 's and 6 's and Case 1 does not hold.

In that case we have infinitely many pairs $x_{i}=6, x_{i+1}=0$. Let $x_{i_{j}}=$ $6, x_{i_{j}+1}=0$ for $j=1,2, \ldots$ and $i_{j}>n$.

We define numbers $y=0 . y_{1} y_{2} \ldots$ and $z=0 . z_{1} z_{2} \ldots$

$y_{i}=x_{i}, z_{i}=x_{i}$ for $i \leq n$,

$\left(y_{i_{j}}=6\right.$ and $\left.y_{i_{j}+1}=0\right)$ or $\left(y_{i_{j}}=5\right.$ and $\left.y_{i_{j}+1}=1\right)$,

$z_{i_{j}}=6, z_{i_{j}+1}=0$ if $y_{i_{j}+1}=0$ and $z_{i_{j}+1}=5$ if $y_{i_{j}+1}=1$,

$y_{i}=2$ if $x_{i}=3, i>n, z_{i}=4$ if $x_{i}=3, i>n$,

$y_{i}=x_{i}, z_{i}=x_{i}$ in other places.

We may easily check that $\frac{y+z}{2}=x$ (since $\frac{y_{i}+z_{i}}{2}=x_{i}$ ), y's and $z$ 's are in $U$ and in $C_{x_{1} x_{2} \ldots x_{n}}$. It's also clear that $y$ 's and $z$ 's form a perfect set.

Case 3. In $x_{1} x_{2} \ldots$ all $x_{i}$ are eventually equal 0 .

Now $x=0 . x_{1} x_{2} \ldots x_{k} 000 \ldots$ and $x_{k} \neq 0$.

If $k>n$ then the set $C_{x_{1} x_{2} \ldots\left(x_{k}-1\right)} \cup C_{x_{1} x_{2} \ldots x_{k}}$ is a desired set. 
If $k \leq n$ then the set $C_{x_{1} x_{2} \ldots\left(x_{k}-1\right) 66 \ldots 6} \cup C_{x_{1} x_{2} \ldots x_{k} 00 \ldots 0}(n-k 0$ 's and $n-k$ 6 's) is a desired set.

Now if we have a copy of $\hat{C}$ in every interval $[n, n+1](n \in \mathbb{Z})$ that is if

$$
C=\bigcup_{n \in \mathbb{Z}} \hat{C}+n
$$

then the theorem 9 holds for any $x \in \mathbb{R}$, and we have the corollary.

Corollary 10. The set $C$ is of measure zero and first category and for every $x \in \mathbb{R}$ and every open neighbourhood $U$ of $x$ there is a perfect set $P$ contained in $C \cap U$ and symmetric about $x$.

In theorem 7 we built a function with infinitely many values. An interesting problem that Ciesielski and Larson ask in [3] is whether this is necessary, that is, if there is a nowhere weakly symmetrically continuous function with finitely many values.

In case of 2-value function we have the following result. (Compare also [3].)

Theorem 11. (Nowik [7]) Every 2-valued function has only countably many points of weak symmetric discontinuity.

Ciesielski in [1] showed also that every 3-valued function has a point of weak symmetric continuity. We do not know if the same is true for 4 -valued functions. Recently Ciesielski and Shelah [4] showed that there is an everywhere weakly symmetrically discontinuous function with bounded range.

\section{References}

[1] K. Ciesielski, On range of uniformly antisymmetric functions, Real Anal. Exchange 19 (1993-94), 616-619.

[2] K. Ciesielski, Set Theory for the Working Mathematician, London Math. Soc. Student Texts 39, Cambridge Univ. Press 1997.

[3] K. Ciesielski, L. Larson, Uniformly antisymmetric functions, Real Anal. Exchange 19(1) (1993-94), 226-235.

[4] K. Ciesielski, S. Shelah, Uniformly antisymmetric function with bounded range, Real Anal. Exchange, to appear.

[5] J. Jaskuła, B. Szkopińska, On the set of points of symmetric continuity, An. Univ. Bucuresti Mat. 37 (1988), 29-35. 
[6] K. Kuratowski, Topology, Vol. I, Acad. Press, New York, N.Y., 1966.

[7] A. Nowik, private communication.

[8] B. S. Thomson, Symmetric properties of real functions, Marcel Dekker, 1994.

[9] B. S. Thomson, Real Functions, Springer-Verlag, 1985. 